\title{
Germanica
}

\section{Zeichen an der Wand. Marlen Haushofers Roman und seine Leser}

Le mur invisible. Le roman de Marlen Haushofer et ses lecteurs

\section{Klaus Haberkamm}

\section{Q OpenEdition}

\section{Journals}

Édition électronique

URL : http://journals.openedition.org/germanica/2177

DOI : 10.4000/germanica. 2177

ISSN : 2107-0784

Éditeur

Université de Lille

\section{Édition imprimée}

Date de publication : 31 décembre 1993

Pagination : 147-162

ISSN : 0984-2632

\section{Référence électronique}

Klaus Haberkamm, «Zeichen an der Wand. Marlen Haushofers Roman und seine Leser », Germanica

[Online], 13 | 1993, Online erschienen am: 10 Februar 2014, abgerufen am 06 Oktober 2020. URL : http://journals.openedition.org/germanica/2177 ; DOI : https://doi.org/10.4000/germanica.2177

Ce document a été généré automatiquement le 6 octobre 2020.

(C) Tous droits réservés 


\title{
Zeichen an der Wand. Marlen Haushofers Roman und seine Leser
}

\author{
Le mur invisible. Le roman de Marlen Haushofer et ses lecteurs
}

\author{
Klaus Haberkamm
}

1 «Habent sua fata libelli», und zwar, wie die vielzitierte Wendung hexametergerecht vervollständigt werden will, «pro captu lectoris» ${ }^{1}$. Marlen Haushofers bedeutendster Roman, Die Wand, bestätigt die Gültigkeit gerade des kompletten Wortlauts noch nach fast zwei Jahrtausenden: An dem Werk demonstriert sich ebenso exemplarisch wie eindringlich schicksalstiftendes Aufnahmeverhalten und Deutungsvermögen - das in der Summe desto unterschiedlicher ausfallen kann, je kürzer, scheinbar weniger differenzbildend der Wirkzeitraum eines Buches ist. Die Wand'bringt es, Phasen des Verstummens eingerechnet, bislang lediglich auf ein Menschenalter; und diese knappe 'Lebenszeit' trägt in der Tat aufschlußreich bei zur Illustration jener Hermeneutik, in deren Sinne die Erkenntnis des Terentianus Maurus hier begriffen ist. Könnte doch die Entfaltung seines Diktums lauten, daß «die individuellen Dispositionen des Lesers, seine Bewußtseinsinhalte, seine epochalen, schichtenspezifisch bedingten Anschauungen sowie seine eigene Erfahrungsgeschichte in mehr oder minder massiver Weise mit den Zeichen des Textes zu einer Sinnkonfiguration zusammengeschlossen werden» ${ }^{2}$. Das «fatum» eines Buches resultiert dabei wesentlich aus der Vervielfältigung des individuellen Lesers zum Kollektiv. Nachdem die Rezeptionstheorie mit Einsichten nach Art des antiken Grammatikers schon sehr früh Kristallisationspunkte gebildet hat, erreicht sie ungefähr mit dem Erscheinen der Wand ihren bisherigen wissenschaftsgeschichtlichen Kulminationspunkt. Diese Koinzidenz legt es nahe, die weit zurückreichende neue Theorie am aktuellen Fall $\mathrm{zu}$ konkretisieren, zumal sich die Tendenzen der Leserreaktion auf diesen Roman für die Gattungsgeschichte der letzten drei Jahrzehnte verallgemeinern lassen.

Bücherschicksal verantworten natürlich nicht zuletzt die professionellen Leser, besonders in Personalunion mit den unbefangenen. Auch der Betrieb der interpretierenden Literaturwissenschaft mit seinen «epistemo- und methodologischen» Vorlieben kann sich immer wieder als Garant für die «Fatalität» 
von Büchern erweisen. Wie Bücher Exempel für bestimmte literarische Richtungen darstellen, so sind sie Demonstrationsobjekte literaturwissenschaftlicher Verfahren; nicht einmal ein Erstsemester der Disziplin wird darin völlig getrennte «fata» erblicken. Aber selbst der fortgeschrittene Studierende, der ein solch enges Junktim wie weiland das zwischen literaturwissenschaftlichem Positivismus und literarischem Naturalismus nun doch nicht mehr erwartet, kann sich gelegentlich eines anderen belehrt finden, Bekanntlich dekretierte ein Großer der Literaturwissenschaft vor rund einem Jahrhundert, daß sich «durchaus lächerlich und verächtlich machen würde», wer «von dem Punkte ab, wo das Dasein von Gespenstern wissenschaftlich widerlegt» sei, einen "Geist aus dem Jenseits» erscheinen ließe, und sei es «zum Zwecke irgendwelcher Aufklärung» ${ }^{3}$. Jene Mimikry angesichts des Knüppels vorgeblicher Lächerlichkeit, die damals die Produktionsanweisung für den «realistischen» Autor forderte, kann zu anderen Zeiten zur Rezeptionsvorschrift für den Interpreten werden. In letzterem Falle wird nicht mehr potentielle Literatur, sondern bereits existente der Wissenschaft unterworfen. Die im Unterschied zur imperialen Ära nicht mehr großmächtige Literaturwissenschaft des demokratischen Zeitalters muß der Literatur den Vortritt lassen, kann auf sie nur reagieren. Dafür übt die Wissenschaft von der Literatur zuweilen kompensatorisch den Gestus der Vorherrschaft gegenüber ihresgleichen, indem sie einzelne literarische Werke, die derartigen Belastungen gar nicht gewachsen sind, für den wissenschaftlichen Verdrängungswettwerb benutzt. Der Preis ist nicht selten deren Beschädigung durch offenkundige philologische Unzulänglichkeit. Kann es zum Trost gereichen, daß auch die Aussparung strukturell relevanter Aspekte bei der Erschließung eines Werkes letztlich Deutungsgeschichte schreibt und indirekt seiner Charakteristik und Zuordnung dient? Schicksale der Bücher aus der Fassungskraft des Lesers ex negativo!

31963 erschienen $^{4}$, erlebte Marlen Haushofers Die Wand fünf Jahre später eine Neuauflage. Trotz dieses bescheidenen Anfangserfolges konnte sich der Roman - der Verlagswechsel mag bereits als Krisenzeichen betrachtet werden - auf Dauer nicht in der Gunst der Leserschaft behaupten. Diese Situation änderte sich mit Beginn der achtziger Jahre. Das, wie es rund anderthalb Jahrzehnte geschienen hatte, tote Buch wurde wiederbelebt und hat sich von da an geradezu eines Booms beim Publikum erfreuen können: Seit der Wiederaufnahme des Werkes in das Programm desjenigen Verlages, der es zuvor abschreiben mußte, folgten nach einem Fortsetzungsdruck in einer überregionalen Tageszeitung bei wechselnden Verlagen mehrere Auflagen, die sich teilweise an spezielle Zielgruppen wie Frauen wendeten. Indiz endgültiger Arriviertheit des Buches beim breiten Lesepublikum war eine Ausgabe speziell für Schüler ${ }^{5}$. Außerdem gelangte Die Wand auf die Leselisten des Goethe-Instituts ${ }^{6}$ und eröffnete sich damit die Chance, den nichtdeutschsprachigen Kulturraum zu erobern.

Der Tod Marlen Haushofers 1970 hatte mit dem vorläufigen Abbruch der Rezeptionsgeschichte des Romans wohl wenig zu tun; eher hätte er zur Förderung des Werkes vermarktet werden können. Ebensowenig reicht das Engagement der Erben der Autorin als Erklärung für die Erneuerung des öffentlichen Interesses an der Wand aus. Das Todesjahr der Haushof er fiel in den gesellschaftlich-geistigen Umbruch nicht zuletzt im deutschsprachigen Raum, der auch dort, wo er nicht ein weiteresmal das Ende der Kunst, insbesondere der Literatur, verkündete, deren Verbreitung nicht in jedem Falle günstig war. Der Erinnerungsbericht einer anonymen Frau in den Vierzigern, die eine plötzliche, unerklärliche Weltkatastrophe als zunächst einzigen überlebenden Menschen in einer nunmehr von einer unsichtbaren, transparenten 
Wand umgebenen Gebirgslandschaft zurückläßt, paßte nach Stoff, Thema und fast Stifterschem Erzählstil nicht zum neuen Zeitgeist - oder schien nicht dazu zu passen. Die allenthalben unter der Jugend um sich greifende Aufbruchstimmung, die nicht nur an der althergebrachten Ordinarienuniversität den "Muff von tausend Jahren» beseitigen wollte, mußte einen scheinbar introvertierten Roman der Endzeit-Kategorie mit seiner notgedrungen individualistischen Protagonistin, deren aufgezwungenes Aussteigertum so gar nicht dem aktuellen Bürgerschreck-Verhalten ähnelte, übersehen. Und wenn damals der Slogan forderte, keinem über dreißig, ob real oder fiktiv, zu trauen, was hätte man dann mit einer alternden, eigentlich uralten Figur und deren noch älteren Verfasserin zu schaffen haben können?! Mit dem Jugendkult um Ulrich Plenzdorf und seinen Edgar Wibeau dann konnten diese schon gar nicht konkurrieren. Gab es überdies nicht genügend Nachholbedarf an Lektüre zu decken, etwa im Bereich der marxistisch-leninistischen Welt-Klassiker, als daß man sich um ein unaufdringliches Werk aus der österreichischen Provinz hätte kümmern können? So mußte die Brisanz des vordergründig idyllischen Romans, der mit seinem vertrackt schlichten Erzählverfahren das Seine dazu tut, das latente Grauen über weite Strecken nicht überhandnehmen zu lassen, vielen potentiellen Lesern entgehen. Muße und Bereitschaft zum genauen Hinsehen fehlten in jener Periode der Aufgeregtheit und der ready-made-Urteile oft allzu sehr. Vielleicht hätte seinerzeit nonkonformistische, sich dem Oktroi des politisierten literarischen Kanons entziehende und sensible Kenntnisnahme das Protestpotential der Wand zum Vorteil der eigenen geschichtlichen Situation erkennen und nutzen können, lange vor den feministischen Töchtern der achtundsechziger Bewegung. Der den makabren Hintergrund des Romangeschehens abgebende Holocaust - eine der von der «Heldin» selbst erwogenen Erklärungsmöglichkeiten - hätte eigentlich ausreichenden Anlaß geboten, die Wand in die heftige Diskussion auch in Deutschland über den noch immer eskalierenden Vietnam-Krieg einzubeziehen. Sollte mangelnder Mut, sich der radikalen Utopie des Romans mit dem Risiko zu stellen, den eigenen, «vorwärtsgewandten» Utopie-Begriff revidieren zu müssen, ein Motiv für sein Ignorieren gewesen sein? Noch ein Jahrzehnt später war Christa Wolfs Erzählung mit dem sprechenden Titel Kein Ort. Nirgends für manchen Eingeschworenen, dem der Gehalt an «ideologiekritischer» Gesellschaftsutopie das zentrale ästhetische Kriterium war, ärgerliche Herausforderung genug.

5 Die Vorlaufzeit seit 1963 reichte der Zweitauflage der Verhalten für sozialen Wandel plädierenden Wand offenbar nicht aus, sich in turbulenten Zeitläuften mit tiefgreifendem Neuerungsprogramm zu behaupten. Dabei galt: «Das Buch ist, wie fast alles, was Marlen Haushof er geschrieben hat, aktuell geblieben - und es ist politisch; beides Einschätzungen, die wir vor zwanzig Jahren wohl entschieden bestritten hätten» ${ }^{7}$. Sicherlich war - gemäß derselben Beurteilung - Haushofers Æuvre im Gegensatz zu dem anderer deutschsprachiger Autoren der Nachkriegszeit «querständig gegen die avantgardistische Moderne». Und doch könnte das Kafkaeske der Wand Indiz für Aneignung der Moderne und eine der Begründungen für den Anfangserfolg des Buches im Horizont der Rezeption Kafkas nach dem Ende von dessen Verfemung sein. Jedenfalls war der Roman von Anfang an so aktuell, wie er es nach 1968 hätte bleiben sollen. Sein apokalyptisches Scenario ließ für die ersten Leser den Abstand knapp zweier Jahrzehnte von der verheerenden Katastrophe des Weltkrieges und speziell den ersten Atombombenabwürfen dahinschrumpfen, zumal die Nachkriegszeit im Zeichen des Kalten Krieges mit 'Höhepunkten' wie dem Korea-Konflikt, den explosiven 
Auseinandersetzungen um Berlin und der Fortentwicklung der Bombe das Bewußtsein einer neuen Ära nicht recht aufkommen ließ. Ein Jahr vor Erscheinen der Wand stand die Welt die brisante KubaKrise durch, die einen globalen Krieg mit extremen Folgen auszulösen drohte. Daß sie zu Beginn des Erscheinungsjahres des Romans offiziell beigelegt wurde, minderte nicht dessen makabre politische Aktualität. Eher wurde der Entspannungsgewinn durch die geopolitische Verunsicherung, die von der Ermordung des amerikanischen Präsidenten noch gegen Ende desselben Jahres ausging, wieder aufgezehrt. Die Leser-Generation in Österreich und Deutschland, die sich in der Nachkriegszeit unter dem Damokles-Schwert jederzeit drohender universeller Vernichtung der Schaffung und Sicherung des «Wirtschaftswunders» widmete, mußte ihr Lebensgefühl in dem Roman parabolisch reflektiert sehen. Zwar wäre es verfehlt, die in der Wand dargestellte agrarische Existenz bei absolutem Neuanfang irgendwie mit dem Morgenthau-Plan in Verbindung zu bringen; doch ließ sich im anheimelnden Detailrealismus des Erzählten das Sinnbild des quietistischen Klimas der restaurativen Nachkriegszeit erkennen. Die Spannung des Romans zwischen Idylle und Apokalypse, eines seiner spezifischen Strukturmerkmale, war die literarische Signatur der Situation Westeuropas und besonders Deutschlands in der Ära des Kalten Krieges, wobei der Nachvollzug der Kessel-atmosphäre des Romans kurze Zeit nach Errichtung der Berliner Mauer als der Trennlinie zwischen zwei diametral entgegengesetzten Welten erleichtert wurde. Sofern die achtundsechziger Bewegung sozialistisch orientiert war, mußte ihr unbeschadet ihrer Vorliebe für die chinesische Kommunismus-Variante eine solche Assoziation ungelegen kommen, geschah also die Vernachlässigung der Wand nicht nur aus Unkenntnis des Buches.

6 Nicht so sehr die Zeitgenossen der Neutronenbombe, deren spezifische Wirkung der Roman Marlen Haushofers vorwegzunehmen scheint, oder die Nachfahren der «Blumenkinder», die in ihm eine Affinität zur Aussteigerkultur ausmachen wollten, verhalfen der Wand in den achtziger Jahren zur Wiederentdeckung. Auch nicht die Leser, die sich dem Werk aus Übersättigung durch eine als einseitig ideologiekritisch empfundene «progressive» Literatur zuwandten, waren hierfür wesentlich. Die Wiederentdeckung verdankt sich vielmehr dem Feminismus, insbesondere der sich zunehmend profilierenden feministischen Literaturwissenschaft, die - exemplarischer Fall des Zusammenspiels von literarischer Intention und Kritik - die Wand als Paradigma der Frauenliteratur ausrief. Dies kam einer späten Rehabilitation des Buches durch die Achtundsechziger gleich. Aufgenommen wurde diese Einschätzung von außerakademischen Emanzipationsbestrebungen, denen der Roman einer, wie es schien, selbstbestimmten Autorin und einer im Wortsinne absoluten Protagonistin als erstaunliches Erbe der patriarchalischen sechziger Jahre in den Blick geraten war. Allerdings boten sich, der nicht mehr homogenen Verfassung des geistigen Klimas zu Beginn der achtziger Jahre entsprechend, die Kräfte der Reaktivierung der Wand gemischt dar. So erspürte man in Assimilation an den Geist seiner konservativen Entstehungszeit eine Art chthonischer Grundgestimmtheit des Romans, von der es nicht weit zu seiner mythologischen Auslegung war. Zur Irritation dezidierter Feministinnen entging die 'Heldin' nicht der mehr oder weniger gewaltsamen Analogisierung mit antiken Göttinnen - war das doch weniger Apotheose der Frau als ahistorische Festschreibung ihrer Verhaltensmuster. 1983, nicht etwa zwanzig Jahre zuvor, erblickte eine Rezension des Buches in der Protagonistin die «lebenspendende Mutter Erde, von deren Mythos wir nie weit entfernt sind $»^{8}$. Mehr noch befremdete Feministinnen, daß überraschend viele Frauen diese Auslegungsrichtung 
mitkonstituierten und, wenn nicht der Mythisierung des Werkes, so dem Rückfall in ihrer Meinung nach geschlechtsspezifische Klischees und Rollenzuweisungen Vorschub leisteten. Es zeigte sich für manche überraschend, daß auch Interpretationen, nicht nur «literarische Produktionen von Frauen an der Geschichte der Weiblichkeitsmythen teilhaben» ${ }^{9}$.

Diejenige Szene des Romans, die strukturell - dem zweiten Brennpunkt einer Ellipse vergleichbar - das Pendant zur plötzlichen Entdeckung der Wand darstellt, wurde als essentielles Moment für den feministischen Gehalt des Werkes ausgegeben: Auftritt und Tötung des mutmaßlich letzten lebenden Mannes. Zwei Jahre nach dem Erscheinen von Günter Grass' Roman Der Butt mit seinem blutigen Vatertags-Kapitel sah man pointiert die «Separation der nicht koexistenzfähigen männlichen und weiblichen Welt und eine so entstehende autonome weibliche Sphäre» als Voraussetzung an zur «Domination durch die Frau», die «idealerweise mehr als Emanzipation» sei. Dementsprechend sei die Heldin (!) in «dieser Einsamkeit als Matriarchin der ihr verbliebenen Lebewesen [...] imstande, ihr in der zivilisierten Welt verkümmertes Potential von Kraft, Geschicklichkeit und Selbständigkeit frei zu entfalten und den ihr gemäßen Vorstellungen zu leben». Dafür wird einiges in Kauf genommen, obgleich unter Utopie-Vorbehalt: «Die Trennung der Geschlechter, und, wenn nötig, die Vernichtung der männlichen Welt und als Konsequenz davon, der gesamten Menschheit, ist in Haushofers Werk eine utopische Lösung, die in dem Roman Die Wand vorgeführt wird $\aleph^{10}$. Fast wirken diese Zuspitzungen wie ein Ausgleich für den Schönheitsfehler, daß der Titel der Wand nach dem Modell der Judenbuche von einem Mann stammt ${ }^{11}$. Die Aggressivität dieser radikalen Position war von sozialkritischem, vermeintlich geschichtsmächtigem Pioniergeist und nicht nur von der Nötigung zur Kompromißlosigkeit im Geschlechterkampf um die Durchsetzung der feministischen Anschauungen bedingt. Sie ließ die Elemente des Romans außer acht, die nicht so sehr für die Darstellung des Loses einer Frau, einer im Sinne dieser Anschauungen glücklichen gar, wie das eines Menschen schlechthin sprechen, und konnte in dieser Form nicht durchgehalten werden. "Der Antizipationsversuch einer möglichen weiblichen Identität, die nicht mehr durch die Existenzform als "anderes Geschlecht" geprägt ist, gibt Haushofers Überlebensmodell jenseits der Zivilisation seine utopische Richtung», wurde in Anspielung an Simone de Beauvoirs Kultbuch behutsamer konstatiert ${ }^{12}$. Vorerst aber noch machte die feministische Forschung beispielsweise den «Umgang weiblicher Autoren mit männlich geprägten Literaturformen» zu ihrem Gegenstand und definierte konkret die Protagonistin der Wand als weiblichen Robinson $^{13}$. Diese trägt schließlich nicht nur aus praktischen Gründen die Kleider ihres Vetters, was sie in der Parabel der Symbolik des Nicht -nur-Weiblichen unterstellt, sondern kann auch gelegentlich die weiterhin virulente Geschlechterspannung nur in dem Wunschtraum aufheben, in die Natur einzugehen. Ausgetragen ist diese Spannung dann, wenn die Erzählerin für die Menschheit, ja für die Lebewesen im ganzen steht, so in dem kaum beachteten Bild, das die seinerzeit populäre Perspektive Camus' auf den SysiphusMythos umkehrt: «Ich konnte zwar wählen, aber nur mit dem Kopf, und das war für mich so gut wie gar nicht. Die Katze und ich, wir waren aus demselben Stoff gemacht, und wir saßen im gleichen Boot, das mit allem, was da lebte, auf die großen dunklen Fälle zutrieb. Als Mensch hatte ich nur die Ehre, dies zu erkennen, ohne etwas dagegen unternehmen zu können. Ein zweifelhaftes Geschenk der Natur [...]» (S. 166). Durch die Ausklammerung solcher Passagen aus der Betrachtung drohen nicht nur die konkreten Frauen, auch die fiktionalen, hinter dem Prinzip des Weiblichen bis zur 
Unkenntlichkeit zurückzutreten - wie es die feministische Literaturwissenschaft pro domo trotz des großen Gewichts der biographischen Methode generell befürchtet - ; unzureichende Differenzierung verzögert auch Einsichten wie die von der «vorfeministischen» Prägung Marlen Haushofers: «Während der Entwurf der neuen "Frauenliteratur" an die Vorstellungen vom Aufbruch zu einer autonomen weiblichen Identität gebunden war, handelt jene, damals von der Frauenbewegung nicht beachtete, heute besonders intensiv rezipierte Literatur von Bachmann, Unica Zürn, Marlen Haushofer und anderen, die Ende der sechziger, Anfang der siebziger Jahre erschienen war, eher von den Kränkungen und Schädigungen weiblicher Existenz und von deren Verstrickungen in die Muster und Mythen von "Weiblichkeit" und weiblicher Sexualität» ${ }^{14}$. Allerdings könnte es angesichts eines antisubjektivistischen Trends in der Literaturwissenschaft überhaupt sinnlos sein, «sich in dem Moment um die weibliche Variante einer Instanz zu bemühen, da diese als allgemeine obsolet geworden zu sein scheint» ${ }^{15}$.

8 Daß die postmoderne Literatur in den sechziger Jahren beginnt, macht noch nicht die Wand $z u$ einem Werk der Postmoderne. Der Gattung anzugehören, die auf Grund ihrer hohen Flexibilität im spielerischen Umgang mit der Welt als die postmoderne schlechthin gilt, kann dafür nicht ausreichen. Zum einen dauert es lange, bis der deutschsprachige Roman Anschluß an die von Amerika, auch Lateinamerika, ausgehende neue Orientierung gewinnt. Zum anderen lehrt, unabhängig von der Chronologie, der bloße Blick auf Beispiele des postmodernen Romans wie Raymond Federmans Alles oder Nichts, Milorad Pavics «Lexikonroman in 100000 Worten», Das Chasarische Wörterbuch, mit seinem weiblichen und männlichen Exemplar oder Italo Calvinos Wenn ein Reisender in einer Winternacht, daß Marlen Haushofers Roman kaum in diese Gruppe einbezogen werden kann. Auch in Burroughs oder Borges - übrigens kaum viel ältere - künstlerische Ahnherrn der Autorin zu sehen dürfte schwerfallen. Konkret genügt die Wand keinem der bislang etablierten Kriterien des postmodernen Romans, mögen es nach Lage der Dinge auch erst wenige sein. Weder verfügt offenbar das Werk über die Leichtigkeit des Spielerischen, die sich - um den Preis des Vorwurfs des Frivolen oder Reaktionären - aus der Verdrängung der Sinnschwere bestenfalls an die Peripherie ergibt. Noch verleiht der «Bericht» dem Leser - wenn es diesen dem apokalyptischen Sujet zum Trotz überhaupt noch gibt - das Privileg, Primus inter pares der am Hervorbringen der ästhetischen Struktur Beteiligten zu sein. Wie ließe sich demzufolge der nur scheinbar offene Schluß des «Berichts» postmodernegerecht in gattungssprengender Fluktuation durch ständige Variation auflösen, mit der nach seiner Logik eigentlich nicht mehr existenten Lebenswelt als Zeichensystem umfassend in ein Austauschverhältnis setzen ? Welche Krisen auch immer die Erzählerin der Wand haben mag - von denen der Zentralfiguren in Paul Austers New-York-Trilogie etwa unterscheiden sie sich wesentlich dadurch, daß sie, wie kompliziert es auch sei, letztlich am Begriff der Identität festhalten.

9 Allerdings lehrt Ecos Im Namen der Rose wenigstens von der herkömmlichen Erzähl weise der Vordergrundshandlung her, daß zum Spiel des postmodernen Romans auch das Auftreten in altmodischer Verkleidung gehören kann. Und der Bannstrahl aus Habermas' linker Hand gegen die aus seiner Sicht neokonservative postmoderne Literatur ließe sich, ungeachtet der petitio principii, auch durch den Verweis auf die kommunikationslose, 'monolektische' Scheinidylle der Wand rechtfertigen. In der Tat hat die poststrukturalistische Forschung sie - der Roman und seine Leser! - als postmodernes Werk entdeckt. Damit haben Feminismus und Dekonstruktion, jene in 
unterschiedliche Richtung gegangenen Ausläufer der achtundsechziger Bewegung, den Roman Marlen Haushofers je zum Demonstrationsfall erhoben und untereinander konfliktträchtige Resultate erzielt. Jene Sorge der feministischen Literaturwissenschaft, mit dem Plädoyer für das emanzipierte weibliche subjekt methodologische Rückständigkeit in Kauf zu nehmen, belegt die Situation der Konkurrenten exemplarisch von der einen Seite - wie von der anderen die gezielte Mahnung der Dekonstruktion, die ästhetische Komplexität der in Frage stehenden Werke transzendiere jede ausschließlich soziale oder politische Fragestellung. Das Augenmerk beider «Parteien» gilt im Falle der Wand dem Exzeptionellen: der absolut auf sich gestellten Frau dort, der «Zone» hier ${ }^{16}$. Diese hermetische «enclosure» ist wie in einigen von Wolfgang Hildesheimers Lieblosen Legenden, in Entropy aus Thomas Pynchons Sammlung Slow Learner oder in John Fowles' Mantissa im poststrukturalistischen Sinne definiert als selbständige Welt, "set off from the exterior world by an ontological borderline» ${ }^{17}$. Erscheint dem postmodernistisch weniger Versierten dieser «Topos» als verwandt mit dem althergebrachten hortus conclusus oder einem ähnlichen enklavenartigen locus amoenus, so nähert er sich immerhin der hier so wichtigen allegorischen Perspektive auf den scheinbar rein realistischen Text. Denn da aus der Sicht postmoderner Ästhetik das immer mögliche Armageddon die Transzendenz ersetzt hat, bedarf es der kompensierenden Allegorie ${ }^{18}$. Wie die Grenze die realistische von der allegorischen Textebene trennt, so markiert sie die vom Poststrukturalismus geförderte Ablösung des Logozentrismus durch den Mythos, der mithin, wo er zum Thema der Wand erhoben wird, nicht von ungefähr die feministische Schule provoziert. Die Grenze stellt damit die Trennlinie dar zwischen unterschiedlichen Erkenntnismöglichkeiten, aber auch Seinsweisen, denen verschiedene Formen des Zeitbewußtseins bis hin zur Zeitlosigkeit, die sich in der Dehnung des Romans spiegele, korrespondieren. Allgemeiner noch markiert die Wand, deren Unsichtbarkeit die Selbstaufhebung der realistischen Sprache des Romans im Wege der Allegorisierung meine, die Grenzen des menschlichen Wissens überhaupt. Sosehr die Wand ontische Bereiche trennt, so sehr muß sie überspielt werden, soll der Leser sich dem Text angemessen nähern. Zunächst einmal kann es für die von der Apokalypse Berichtende als einzige Überlebende keine Leser mehr geben, und doch benötigt sie nach eigenem Bekunden die Vorstellung, ihren Bericht für Rezipienten zu verfassen. Gibt es aber Leser, hebt sich zusammen mit seiner Prämisse der Bericht in seinem Wahrheitsanspruch auf, wie umgekehrt mit dessen Stimmigkeit sein Leser als romanimmanenter $\mathrm{zu}$ existieren aufhört. Beides zugleich, typisch postmodern, ist notwendig und möglich. Der tötende Eindringling, der getötet wird, läßt sich gerade auf Grund der «Sprachlosigkeit» des Vorgangs als Allegorie dieses ständigen Wechselspiels der Nichtkommunikation interpretieren. Angemessene Lektüre würde folglich nicht durch papiervermittelte Repräsentation des Geschehens ermöglicht, sondern durch direkte Präsentation, wie es die Erzählerin selbst erwägt: «Vielleicht müßte ich [...] mit Kieselsteinen auf grünes Moos zeichnen oder mit einem Stock in den Schnee ritzen» (S. 194). Somit entgeht nur derjenige Leser, der die «Menschenschrift» (S. 194) als Allegorie «durchschaut», der Gefahr, dem Text Gewalt anzutun oder von ihm «eliminiert» zu werden. "Writing with stick and stone would unify writer and reader in the same space. Reading the narrator's ideal and most real text, carved int $o$ the surface of her isolated world, would be reading her world within her enclosure instead of a "Menschentext" on paper about (and outside of) her isolated world. Reading her carved enclosure as a text, then, would mean sharing her fate not as a witness 
reading in an easy chair but as a fellow victim of the catastrophe» ${ }^{19}$. Damit wäre aber eine letzte, methodologische Grenze vom poststrukturalistischen Verfahren überschritten: Der mit dem «Bericht» konstituierte Leser, der zumindest als potentieller immer schon immanenter ist, würde sich unversehens in den höchst realen externen des Romans verwandeln, dem in seinem Lesesessel die Seinsbereiche von Realität und Fiktion verschwämmen, der sich selbst zur Fiktion würde...

«Ich schreibe nicht aus Freude am Schreiben», beginnt die Erzählerin der Wand ihren Bericht, "es hat sich eben so ergeben, daß ich schreiben muß, wenn ich nicht den Verstand verlieren will» (S.7). Es geht ihr - wie manchen ihrer modernen Leidensgenossinnen und-genossen in litteris und übrigens ihrem idealen postmodernen Leser - um den Schreibprozeß als solchen, nicht um das Ergebnis, das ohnehin zum Mäusefutter bestimmt ist. Schon zu Beginn der Niederschrift wird die Frau von der Angst beherrscht, am Tier vorbei in den Abgrund zu stürzen. Marlen Haushofers Erzählerin - ähnlich ihrer Autorin - muß schreiben, will sie Mensch bleiben.

11 Irmgard Morgner, in der DDR Verfasserin von Frauenliteratur und Anwältin der Frauenrechte, betonte dagegen in einem Interview kurz vor ihrem Tod 1990, nicht unbedingt schreiben $\mathrm{zu}$ müssen. "Wozu soll ich mich überhaupt abquälen, die verschleißende Arbeit der Fertigstellung von Texten [...] zu leisten, wenn keiner die Konzentration aufbringt, die meine Texte fordern? $»^{20}$ Die im Zeichen der Vereinigung Deutschlands mit Blick auf die kapitalistische Mediengesellschaft und ihre Unterhaltungsindustrie gestellte Frage gilt - unabhängig vom sozialen System - der gesamten Nachkriegszeit. In ihr klingt die Elegie von der gefährdeten Buchkultur an, deren leiser Gegenstand und subtile Legitimation im Wortsinne übertönt werden von immer neuen Attraktionen und Sensationen. Dem Dementi aus Enttäuschung liegen durchaus der Drang zu schreiben und die Sehnsucht nach Lesern zugrunde; und dieses Begehren ist um so heftiger, je mehr die Schreibende - sei es die Erzählerin der Wand, Marlen Haushofer oder Irmgard Morgner - ausschließlich von sich selbst zu schreiben scheint. Ob wirkliche Person oder fiktionale Figur, sie schreibt im Bewußtsein einer untergehenden Kultur, ohne Hoffnung auf den Leser, für sich allein. Insofern bringt Die Wand ein spätzeitliches Krisenbewußtsein, ein kulturpessimistisches Lebensgefühl aus der Sicht von Schreibenden gleichnishaft zum Ausdruck. Diese Befindlichkeit ist in Deutschland besonders nach der Vereinigung zutage getreten, die ihrerseits im Zusammenhang mit dem Kollaps der materiellen und ideellen Weltordnung der Ära nach 1945, damit auch von Feindbildern und Lebenslügen wie bei einigen SpätAchtundsechzigern, zu sehen ist. Die resigniert-tapfere Feststellung der todgeweihten Irmgard Morgner trifft schon auf die Situationen ihrer zwanzig Jahre zuvor verstorbenen Schicksalsgenossin Marlen Haushofer und von deren Protagonistin zu: "Am Ende bleibt doch das eigene Leben». Wie wenig diese Gestimmtheit auf schreibende Frauen beschränkt ist, zeigt sich etwa an jüngsten Erkenntnissen Bodo Kirchhoffs.

12 Für Kirchhoff sind die Schriftsteller mit dem Zusammenbruch des Sozialismus zu «Kollegen in der Ohnmacht» ${ }^{21}$, ohne Einfluß mehr auf die öffentliche Meinung, geworden. Sein verbleibendes Schreibmotiv, «ein Leiden oder Unbehagen an mir zu mildern, mich also durch Schreiben besser zu fühlen», ist bereits das Marlen Haushofers und ihrer Protagonistin, auch wenn Kirchhoff sich nicht als Autor von «Einsamkeit, Armut, Wahnsinn» verstehen will. Vor der Darstellung bloßer Ichhaftigkeit schütze ihn nichts mehr. Was für die Erzählerin der Wand gewissermaßen 
nach einer Epochenzäsur absolute Notwendigkeit ist, fällt dem Zeugen von 1968 an einer neuerlichen Zeitenwende sichtlich schwer. Das berühmte Bekenntnis des Erzählers von Max Frischs Gantenbein zu den Ich-Geschichten auch in äußersten sozialen Turbulenzen geht ihm nur mühsam über die Lippen, doch läßt ihm die «Zeit der Formlosigkeit», das Fehlen einer verbindlichen geistigen Aufgabe für den Schriftsteller, keine Wahl. In Wirklichkeit aber ist der «Rückzug» des Erzählens ,auf das Leiden, das «Abwandern in die Allegorie», wie es mit Seitenhieb auf die postmoderne Literatur heißt, nicht erst von der akuten Lage der Gesellschaft erzwungen, wie es die Wand eine Generation früher belegt. Deren Aktualität, wie die des gesamten Euvres Marlen Haushofers, bestätigt sich im literarischen Programm Kirchhoffs: «Wenn ich nicht schweigen will oder nur von den Schwierigkeiten des Schreibens erzählen, muß ich ausziehen und Gleichnisse auf mein Leben erfinden, gestützt auf die zwei einzigen, in ihrer ganzen Fülle und Grausamkeit unangreifbaren Wahrheiten, Liebe und Tod [...]» Kirchhoffs Ausrichtung auf die «Wüste des Banalen», die den Existenzvorsprung des Autors und damit seine Glaubwürdigkeit verbürge, und sein antipostmodernistischer Sarkasmus, nur noch darüber schreiben zu können, «daß man eigentlich nicht schreiben kann, es aber dennoch tut und weiß, daß man es dennoch tut, und weiß, daß man weiß, daß man es dennoch tut», implizieren letztlich die Anerkennung der Erzählweise der scheinbar schlichten, ephemeren Wand. Wer sich wie Kirchhoff als Schriftsteller der Gegenwart mit dem Grauen des Trivialen konfrontiert sieht und nur noch über die Flucht davor authentisch schreiben zu können meint, findet in Marlen Haushofers Roman von 1963 ein immer noch und immer wieder gültiges Modell. «Vielleicht», bietet die Erzählerin, die in ihrem Leben vor der Katastrophe «die Langeweile eines biederen Rosenzüchters auf einem Kongreß der Autofabrikanten» (S.182) empfindet, aber auch später nicht frei ist von dem «Gefühl einer großen Einsamkeit, Leere und Helle» (S. 186), eine bedenkens werte Deutung der Wand an, «war die Wand auch nur der letzte verzweifelte Versuch eines gequälten Menschen, der ausbrechen mußte, ausbrechen oder wahnsinnig werden» (S. 91).

\section{NOTES}

1. - Karl Lachmann (Hg.), Terentiani Mauri de litteris syllabis et metris liber, Berlin 1836, V. 1286.

2. - Wolfgang Iser, «Der Lesevorgang. Eine phänomenologische Perspektive», in Rainer Warning (Hg.), Rezeptionsästhetik. Theorie und Praxis, München 1975 (UTB 303), S. 253-276, hier: S. 264.

3. - Wilhelm Bölsche, Die naturwissenschaftlichen Grundlagen der Poesie. Prolegomena einer realistischen Aesthetik, Leipzig 1887, S. 4f.

4. - Entgegen vor allem im Feuilleton verbreiteten Angaben ist festzustellen, daß Die Wand 1963 (Sigbert Mohn Verlag, Gütersloh), nicht 1968, erstmals veröffentlicht wurde. 1968 brachte der Claassen-Verlag, Hildesheim, die zweite Auflage als «Ciaassen Sonderausgabe» heraus.

5. - Das Profil der «Frankfurter Rundschau», die den Fortsetzungsdruck brachte, ist symptomatisch für die Tendenz der zweiten Rezeptionphase des Romans. - Auf seinen Neudruck in Buchform bei Ciaassen 1983 folgten 1985 bei Ullstein, Berlin/Frankfurt, das Ullstein-Buch Nr. 30169 in der Reihe «Die Frau in der Literatur», 1986 bei Klett, Stuttgart, eine für Schulen 
gedachte Ausgabe mit einem erläuternden Anhang sowie noch 1991 eine Edition beim Deutschen Taschenbuch Verlag, München (dtv Nr. 11607), die bereits in mehreren Auflagen vorliegt. (Nach dieser Ausgabe wird hier, mit dem jeweiligen Seitenbeleg im laufenden Text, zitiert.)

6. - Vgl. Hugo Caviola, «Behind the Transparent Wall. Marlen Haushofer's Novel Die Wand», in MAL 24 (1991), Nr. 1, S. 101-112, hier: S. 111 Anm. 1.

7. - Uwe Schweikert, «Im toten Winkel. Notizen bei der Lektüre von Marlen Haushofers Roman Die Wand», in Anne Duden (Mitverf.): «Oder war da manchmal noch etwas anderes?» Texte zu Marlen Haushofer, Frankfurt/M. 1986, S. 11-20, hier: S. 17.

8. - E.H., «Die vertane Möglichkeit. Marlen Haushofers Roman Die Wand», Neue Zürcher Zeitung, 17.11.1983, S. 36.

9. - Sigrid Weigel, «Geschlechterdifferenz und Literaturwissenschaft», in Helmut Brackert, Jörn Stückrath (Hg.), Literaturwissenschaft. Ein Grundkurs, Reinbek 1992 (re 523), S. 677-689, hier: S. 679.

10. - Dagmar C. G. Lorenz, «Marlen Haushofer - eine Feministin aus Österreich», in MAL 12 (1979), Nr. 3/4, S. 171-191, hier: S. 679.

11. - Auch der Titel der Mansarde geht auf den Förderer und Berater Marlen Haushofers, Hans Weigel, zurück. Vgl. Dagmar C. G. Lorenz, «Marlen Haushofer - eine Feministin aus Österreich», S. 189 Anm. 18.

12. - Isolde Schiffermüller, «Weibliche Utopie und Selbstverleugnung. $\mathrm{Zu}$ den Widersprüchen in Marlen Haushofers Roman Die Wand», in Quaderni di Lingue e Letterature 13 (1988), S. 139-149, hier: S. 142.

13. - Vgl. Irmgard Roebling, «Ist Die Wand von Marlen Haushofer eine weibliche Robinsonade?», in Diskussion Deutsch 20 (1989), S. 48-58, hier: S. 48.

14. - Sigrid Weigel, «Frauenliteratur - Literatur von Frauen», in Klaus Briegleb und Sigrid Weigel (Hgg.); Gegenwartsliteratur seit 1968, München, Wien 1992 (Hansers Sozialgeschichte der deutschen Literatur vom 16 Jahrhundert bis zur Gegenwart, Bd. 12), S. 245-276, hier: S. 248.

15. - Sigrid Weigel, «Geschlechterdifferenz», S. 683.

16. - Vgl. Hugo Caviola, «Behind the Transparent Wall», und ders: In the Zone. Perception and Presentation of Space in German and American Postmodernism, Basel, Boston, Berlin. 1991 (ICSELL).

17. - Caviola, In the Zone, S. 128.

18. - Vgl. Andreas Kilb, «Die allegorische Phantasie: Zur Ästhetik der Postmoderne», in Christa und Peter Bürger (Hgg.): Postmoderne: der Alltag, die Allegorie und die Avantgarde, Frankfurt/M. 1987 (stw 648), S. 84-113, hier: S. 111 - Vgl. auch Caviola, In the Zone, S. 142 und Anm. 22.

19. - Caviola, In the Zone, S. $145 \mathrm{f}$.

20. - Synnöve Clason, «Am Ende bleibt das eigene Leben. Ost-Berlin, 1990: Ein Gespräch mit Irmtraud Morgner - kurz vor ihrem Tod», ZEIT, 6.11.1992, S. 6.

21. - Bodo Kirchhoff, «Das Schreiben: ein Sturz. In der Wüste des Banalen - zur Lage des Schriftstellers in glücklicher Zeit», ZEIT, 6.11.1992, S. 5.

\section{RÉSUMÉS}

Der Beitrag versucht im Sinne des Rahmenthemas dieses Heftes, hervorstechende Rezeptionsweisen des akademischen und nichtakademischen deutschsprachigen Lesepublikums während der letzten drei Jahrzehnte zu skizzieren. Demonstrationsobjekt ist Marlen Haushofers 
bedeutendster Roman, Die Wand (1963). An diesem Paradigma bestätigt sich noch nach fast zwei Jahrtausenden eindringlich die Gültigkeit des in Deutschland üblicherweise verkürzt zitierten Hexameters des Terentianus Maurus: «pro captu lectoris habent sua fata libelli». Der Vers formuliert bereits, scheint es, den Kern der um die Erscheinungszeit des österreichischen Werkes immer stärker hervortretenden Rezeptionstheorie, wobei allerdings der relativierende Einschlag im antiken Reflex auf das «Fassungsvermögen des Lesers» nicht übersehen werden sollte. $\mathrm{Zu}$ solcher Relativierung könnte der Vergleich zwischen den hier in konkreter Illustration der Rezeptionstheorie knapp präsentierten Deutungen desselben Textes (Prespektive des Kalten Krieges, der Studentenbewegung, des Feminismus, der Postmoderne, des Nach-Sozialismus) verleiten, sofern die hermeneutischen Elemente der Geschichtlichkeit bzw. Subjektivität in ihrer Unabdingbarkeit außer acht blieben.

Cette contribution tente d'esquisser, dans l'esprit de la thématique de ce numéro, certains modes de réception remarquables des lecteurs de langue allemande, universitaires ou non, durant les trente dernières années. Le roman le plus important de Marlen Haushofer, Die Wand (1963), constituera l'objet de notre démonstration. Mesuré à ce paradigme, l'hexamètre de Terentianus Maurus, communément abrégé en Allemagne, confirme toute sa pertinence (même après presque deux millénaires) : "pro captu lectoris habent sua fata libelli». Ce vers formule déjà, semble-t-il, le sens profond de la théorie de la réception qui s'affirme de plus en plus nettement au moment de la parution de l'œuvre autrichienne, sans toutefois qu'il faille perdre de vue la relativisation contenue dans le réflexe antique de « l'intelligence du lecteur ». C'est à ce genre de relativisation que pourrait conduire la comparaison entre les interprétations présentées ici de manière volontairement, d'un même texte (perspective de la guerre froide, du mouvement étudiant, du féminisme, de l'épque post-moderne, de l'après-socialisme), destinées à illustrer concrètement la théorie de la réception, dans la mesure où les éléments herméneutiques de l'historicité, ou même de la subjectivité n'ont pas été pris en considération dans leur « inaliénabilité ».

\section{AUTEUR}

\section{KLAUS HABERKAMM}

Westphälische-Wilhelms-Universität Münster 